\title{
Potential protective role of a NOD2 polymorphism in the susceptibility to multiple sclerosis is not associated with interferon therapy
}

\author{
AIDA ZEČKANOVIĆ ${ }^{1}$, ALEŠ MAVER ${ }^{1}$, SMILJANA RISTIĆ$^{2}$, \\ NADA STARČEVIĆ ČIZMAREVIĆ ${ }^{2}$, BORUT PETERLIN ${ }^{1}$ and LUCA LOVREČIĆ ${ }^{1}$ \\ ${ }^{1}$ Clinical Institute of Medical Genetics, University Medical Centre Ljubljana, 1000 Ljubljana, Slovenia; \\ ${ }^{2}$ Department of Medical Biology and Genetics, Faculty of Medicine, University of Rijeka, 51000 Rijeka, Croatia
}

Received February 11, 2021; Accepted May 28, 2021

DOI: $10.3892 / \mathrm{br} .2021 .1476$

\begin{abstract}
Pattern recognition receptors, such as specific nucleotide-binding oligomerization domain protein 2 , and their polymorphisms may be involved in the pathogenesis of multiple sclerosis (MS). They may also play a role in the formation of neutralizing antibodies against interferon- $\beta$ (INF- $\beta$ ), and may exhibit lowered efficacy. Identification of these polymorphisms may be useful for early identification of potential non-responders and to allow for modification of treatment regimens earlier. The differences in genotype distribution and allele frequency of the rs3135499 and rs2066842 NOD2 polymorphisms between patients with MS and healthy controls were analysed in the present study. The group of patients were divided into responders and non-responders to INF- $\beta$ therapy to evaluate the association of both polymorphisms with response to therapy. No differences in the genotype frequencies between the responder and non-responder groups were observed. However, a statistically significant difference in genotype frequencies of TT homozygotes for rs2066842 between patients with MS and healthy controls was observed $\left(\chi^{2}=11.8 ; \mathrm{P}=0.003\right)$. A recessive genotype model and allele distribution in rs2066842 suggest that the genotype TT and allele $\mathrm{T}$ itself are protective against MS. The odds ratio of 0.12
\end{abstract}

Correspondence to: Professor Luca Lovrečić, Clinical Institute of Medical Genetics, University Medical Centre Ljubljana, Šlajmerjeva 4, 1000 Ljubljana, Slovenia

E-mail: lucalovrecic@gmail.com

Abbreviations: MS, multiple sclerosis; RRMS, relapsing/remitting MS; NOD2, specific nucleotide-binding oligomerization domain protein 2 ; PRR, pattern recognition receptor; INF- $\beta$, interferon- $\beta$; $\mathrm{NAb}$, neutralizing antibody; SNP, single nucleotide polymorphism; IL, interleukin; EDSS, expanded disability status score; RAR, retinoic acid receptor; $\mathrm{RXR}$, retinoid $\mathrm{X}$ receptor

Key words: pattern recognition receptor, INF- $\beta$, NAb, multiple sclerosis represents an 8.33x lower risk for MS if an individual has a TT genotype. The significantly lower incidence of the TT genotype of rs2066842 in patients with MS suggests that the TT genotype and $\mathrm{T}$ allele may be a protective genetic factor against MS.

\section{Introduction}

Multiple sclerosis (MS) is an autoimmune inflammatory disorder of the central nervous system caused by the interplay of genetic and environmental factors. It is the most common cause of neurological disability in younger individuals with a lifetime risk of 1 in a 400 that affects women twice as frequently as men. The majority of patients present with the relapsing/remitting (RRMS) form of the disease, which normally has three phases: Relapses with complete recovery, relapses with a persistent disability, and secondary progression (1). The precise etiopathogenetic mechanisms of the disease remain unclear. Several disease-modifying drugs are already available and new promising drugs are emerging; however, interferon- $\beta$ (INF- $\beta$ ) is still the favoured first-line treatment for RRMS $(2,3)$. Nevertheless, a subgroup of patients do not respond to this treatment; only $\sim 60 \%$ of patients on INF- $\beta$ treatment were relapse-free for 2 years (responders), and in $30 \%$ of patients, the disease progressed over 2 years whilst the study was ongoing $(4,5)$. The true therapeutic effects of INF- $\beta$ can only be measured after several months of treatment, which makes management of MS even more challenging (3-5). Surrogate biomarkers for early identification of non-responder patients may enable timely adjustments to the MS therapeutic regimen, and consequently improved outcomes, but no such biomarkers are currently available $(2,6)$. In addition, a considerable proportion of patients with MS (14-22\%) on INF- $\beta$ therapy develop neutralizing antibodies (NAb) against INF- $\beta$, which have been shown to reduce the positive effects of INF- $\beta$ on disease progression $(4,7,8)$. A previous study examined the role of the pattern recognition receptors (PRRs) in facilitating $\mathrm{NAb}$ formation, as PRRs participate in the immune response towards foreign substances (6).

Specific nucleotide-binding oligomerization domain protein 2 (NOD2; OMIM\#, 605956) belongs to the family of PRRs, and specific NOD2 polymorphisms have already been 
investigated with regard to MS. One study observed an association between rs3135499 and time to relapse of MS, as well as rs2066842 and frequency of NAb development. However, these observations were not considered statistically significant after correction for multiple comparisons (6). The association of rs2066842 with the Th17 response to the myelin basic protein in patients with MS has also been suggested, but not confirmed (9). Another study tested multiple polymorphisms in genes of the innate immune system and again found no link between rs2066842 and MS diagnosis (10).

The aim of the present study was to investigate the association between NOD2 gene polymorphisms and susceptibility to $\mathrm{MS}$, as well as the association with response to IFN- $\beta$ treatment in a cohort of Slovenian and Croatian patients with MS who were treated with IFN- $\beta$.

\section{Materials and methods}

Study population. A total of 267 patients with MS (203 females and 64 males; median age at onset, 28 years; age range 14-55 years) treated with IFN- $\beta$ at the University Medical Centre Ljubljana (Ljubljana, Slovenia) and the Clinical Hospital Center Rijeka (Rijeka, Croatia) were included in the present study. All patients were diagnosed prior to enrolment using the McDonald diagnostic criteria (11). Patients with MS and other chronic diseases were excluded. For the evaluation of treatment response (responder/non-responder), clinical status was followed prospectively for at least 2 years following initiation of therapy (12). An exacerbation was defined as new, worsening or recurrent neurological symptoms attributable to MS. A responder was defined as a patient with no MS relapses within the first 2 years after treatment initiation, independent of the Kurtzke's Expanded Disability Status Score (EDSS) (13). After applying the above mentioned criteria, there were 175 responders and 92 non-responders in the group of patients with MS. The group of healthy controls consisted of 234 individuals (17 females and 217 males; median age at onset 40 years; age range 22-68 years) with no neurological disease in their health history. The present study was approved by the Ethical Committees of both participating centres, and all subjects provided informed consent to participate.

Sample collection and selection of single nucleotide polymorphisms (SNPs). Genomic DNA was extracted from peripheral blood leukocytes using a QIAmp blood kit (Qiagen $\mathrm{GmbH}$ ) according to the manufacturer's protocol.

The NOD2 polymorphisms were selected based on previous studies and data (rs3135499 and rs2066842) $(6,9,10)$. Additional information regarding the selected SNPs was obtained using GeneCards (genecards.org), The UCSC Genome Browser, ENSEMBL and Alggen Promo (14-17). Genotyping was performed using the TaqMan ${ }^{\circledR}$ Genotyping assays using allele-specific quantitative PCR and TaqMan ${ }^{\circledR}$ MGB probes, according to the manufacturer's protocol (Applied Biosystems; Thermo Fisher Scientific, Inc.). An ABI PRISM $^{\circledR} 7000$ Sequence Detection system was used for PCR amplification and signal detection, and ABI PRISM ${ }^{\circledR} 7000$ SDS version.1.2 with the RQ Study Results software (Applied Biosystems; Thermo Fisher Scientific, Inc.) was used for data analysis.
Statistical analysis. Genotype frequency data was acquired from 1000 Genomes, Candidate GeneSNPSelection(GenePipe) and Ensembl (accessed on May 2021) (18,19). The association between the genotypes and INF- $\beta$ responder/non-responder status was calculated using a $\chi^{2}$ test or a Fisher's exact test. $\mathrm{P}<0.05$ was considered to indicate a statistically significant difference $(\alpha)$. The Hardy-Weinberg equilibrium equation was used to determine if the sample was representative. Data were analysed using the Court lab-HW (20) calculator and SPSS version 22 (IBM Corp.).

\section{Results}

In the group of 267 patients, there were 204 female and 63 male patients (Table I). Both males and females were distributed evenly amongst the responder and non-responder groups, with females accounting for $76 \%$ of patients with MS, $75 \%$ of the responder group and $78 \%$ of the non-responder group. The observed genotype distribution of rs3135499 and rs2066842 showed no significant differences when compared with those predicted from the Hardy-Weinberg equilibrium for either patients or controls $(\mathrm{P}>0.05)$.

The rs2066842 polymorphism displayed a statistically significant difference in the genotype frequencies in patients with MS, with the TT genotype being significantly less common in the group of patients with MS than expected $\left(\chi^{2}=11.8 ; \mathrm{P}=0.003\right)$. There were no significant deviations in the rs3135499 allele distribution (Table II).

In addition, further statistical analysis for the recessive model and allele distribution of rs2066842 were calculated. The findings suggest that the TT genotype and the $\mathrm{T}$ allele itself are protective against MS (Table III); the TT genotype was associated with an 8.33x lower risk for MS (odds ratio=0.12, $\mathrm{P}=0.0008$ ).

Because of the highly significant difference in genotype frequencies between MS patients and controls the genotype distribution of rs2066842 and rs3135499 were compared between the control group with the data for the European population from the 1000 Genomes database, and found that both distributions were very similar (data not shown) (15).

The comparison of rs3135499 and rs2066842 genotype frequencies between the responder and non-responder groups showed no significant deviations when all patients with MS were grouped together or when stratified by sex (Table IV).

\section{Discussion}

In the present study, two NOD2 polymorphisms and their association with MS were studied. A statistically significant lower frequency of the TT genotype and T allele of rs2066842 in patients with MS was found. It is hypothesized that the $\mathrm{T}$ allele has a protective effect against MS with the carriers of TT being $8.33 x$ less likely to develop MS than the carriers of a CT or CC genotype.

These results do not imply a direct causal relationship, nevertheless rs2066842 may have an influence on susceptibility to MS. No specific mechanism by which the TT genotype or the $\mathrm{T}$ allele may have a protective effect on MS has been studied yet, to the best of our knowledge. In silico predictions for SNP function link rs2066842 to a nonsense-mediated mRNA 
Table I. Clinical characteristics of MS patients.

\begin{tabular}{lcrr}
\hline Chaarcteristics & All & Women & Men \\
\hline Total $(\%)$ & 267 & $204(76 \%)$ & $63(24 \%)$ \\
Age at onset $^{\mathrm{a}}$ & $28.7 \pm 8.1$ & $28.5 \pm 8.4$ & $29.6 \pm 7.0$ \\
Duration,years $^{\mathrm{a}}$ & $9.9 \pm 6.9$ & $10.3 \pm 7.1$ & $8.4 \pm 6.0$ \\
EDSS $^{\mathrm{a}, \mathrm{b}}$ & $3.3 \pm 2.9$ & $3.3 \pm 3.1$ & $3.1 \pm 2.0$ \\
PI $^{\mathrm{a}, \mathrm{c}}$ & $0.38 \pm 0.28$ & $0.36 \pm 0.28$ & $0.42 \pm 0.29$
\end{tabular}

${ }^{\mathrm{a}}$ Mean \pm standard deviation; ${ }^{b}$ Disability was assessed using Kurtzke's EDSS; ${ }^{\mathrm{P} I}$ is expressed as ratio EDSS/disease duration in years. EDSS, Expanded Disability Status Scale; PI, progression index.

Table II. Comparison of genotype frequencies of the rs3135499 and rs206684 polymorphisms between patients with MS and the healthy controls.

\begin{tabular}{|c|c|c|c|c|c|}
\hline Single nucleotide polymorphisms & Genotype & MS, n (\%) & Control, n (\%) & $\chi^{2}$ & $\mathrm{P}$-value \\
\hline \multirow[t]{4}{*}{ rs3135499 } & AA & $64(24.1)$ & $60(26.5)$ & \multirow[t]{4}{*}{$\chi^{2}=1.952$} & \multirow[t]{4}{*}{$\mathrm{P}=0.377$} \\
\hline & $\mathrm{AC}$ & $154(57.9)$ & $117(51.8)$ & & \\
\hline & $\mathrm{CC}$ & $48(18.0)$ & $49(21.7)$ & & \\
\hline & $\Sigma$ & $266(100.0)$ & $226(100.0)$ & & \\
\hline \multirow[t]{4}{*}{ rs2066842 } & TT & $2(0.7)$ & $14(6.0)$ & \multirow[t]{4}{*}{$\chi^{2}=11.8$} & \multirow[t]{4}{*}{$\mathrm{P}=0.003^{\mathrm{a}}$} \\
\hline & $\mathrm{TC}$ & $102(38.2)$ & $93(39.7)$ & & \\
\hline & $\mathrm{CC}$ & $163(61.1)$ & $127(54.3)$ & & \\
\hline & $\Sigma$ & $267(100.0)$ & $234(100.0)$ & & \\
\hline
\end{tabular}

${ }^{\mathrm{a}} \mathrm{P}<0.01$. MS, multiple sclerosis.

Table III. ORs for rs2066842 genotypes and alleles in patients with MS compared with the control subjects.

\begin{tabular}{lcc}
\hline Statistics & TT vs. TC and CC & Allele T vs. Allele C \\
\hline OR (95\% CI) & $0.12(0.03-0.53)$ & $0.71(0.53-0.96)$ \\
P-value & $0.0008^{\mathrm{b}}$ & $0.024^{\mathrm{a}}$ \\
\hline
\end{tabular}

${ }^{\mathrm{a}} \mathrm{P}<0.05,{ }^{\mathrm{b}} \mathrm{P}<0.001$. CI, confidence interval; MS, multiple sclerosis; $\mathrm{OR}$, odds ratio.

decay, a process that influences the amount of transcript and therefore, the amount of protein produced, and its function $(14,15)$. In addition, both E2F-1 and retinoid $\mathrm{X}$ receptor (RXR)- $\alpha$ transcription factors can bind to allele $\mathrm{C}$, but only E2F-1 can bind to the protective allele T (17).

The RXR- $\alpha$ has already been investigated in association with MS. Mean plasma retinol levels in untreated RRMS patients were lower compared with the IFN- $\beta 1$ a treated patients, as well as compared with patients with a non-inflammatory neurological disease. In the IFN- $\beta 1$ a treated patients, the expression of 5 retinoid receptor subtypes was reported (retinoic acid receptor (RAR)- $\alpha$, RAR- $\gamma$, RXR- $\alpha$, RXR- $\beta$ and RXR- $\gamma$ ), whilst only the first three were expressed in untreated RRMS patients. Therefore, the disease activity in MS may be associated with retinol levels, and activation of retinoid receptors may be a mechanism of action of IFN- $\beta$ (21).

A previous study reported no relationship between rs2066842 and MS diagnosis, in contrast with the results of the present study, and this may be due to the heterogeneity of the study group recruited by Enevold et al (10), where all types of patients with MS were included, as opposed to the present study, which only included RRMS patients receiving immunomodulatory treatment.

In addition, the association between NOD2 gene polymorphisms rs2066842 and rs3135499 with response to therapy with IFN- $\beta$ in MS patients were analysed. To the best of our knowledge, this is the first study to do so. No significant differences in genotype frequencies for rs2066842 and rs3135499 between the responder and non-responder groups, as a whole, or when stratified by sex was observed. The highly variable course of MS significantly complicates the prediction of the outcome of IFN- $\beta$ therapy and predictors of response would greatly enhance the management of patients. So far, no such surrogate endpoint exists, to the best of our knowledge. Studies have investigated the role of genetic polymorphisms in NAb formation, mostly in relation to HLA II characteristics, but the results have not been implemented in clinical practice (22-24).

Important insights into the immunopathogenetic concepts of MS have been gained. A study by Arellano et al (25) demonstrated a notable increase in production of Th17 and Th1 lymphocytes and associated cytokines (such as, IFN- $\gamma$ 
Table IV. Comparison of genotype frequency of polymorphisms rs3135499 and rs206684 based on response to treatment and sex.

\begin{tabular}{|c|c|c|c|c|c|c|}
\hline $\begin{array}{l}\text { Single nucleotide } \\
\text { polymorphisms }\end{array}$ & Sex & Genotype & Responders, n (\%) & Non-responders, n (\%) & Statistics & P-value \\
\hline \multirow[t]{12}{*}{ rs3135499 } & All & AA & $43(24.7)$ & $21(22.8)$ & $0.209^{\mathrm{a}}$ & 0.901 \\
\hline & & $\mathrm{AC}$ & 99 (56.9) & $55(59.8)$ & & \\
\hline & & $\mathrm{CC}$ & $32(18.4)$ & $16(17.4)$ & & \\
\hline & & $\Sigma$ & $174(100)$ & $92(100)$ & & \\
\hline & Male & AA & $10(22.7)$ & $7(35)$ & $1.12^{\mathrm{b}}$ & 0.626 \\
\hline & & $\mathrm{AC}$ & $25(56.8)$ & $10(50)$ & & \\
\hline & & $\mathrm{CC}$ & $9(20.5)$ & $3(15)$ & & \\
\hline & & $\Sigma$ & $44(100)$ & $20(100)$ & & \\
\hline & Female & AA & $33(25.4)$ & $14(19.4)$ & $0.951^{\mathrm{a}}$ & 0.622 \\
\hline & & $\mathrm{AC}$ & $74(56.9)$ & $45(62.5)$ & & \\
\hline & & $\mathrm{CC}$ & $23(17.7)$ & $13(18.1)$ & & \\
\hline & & $\Sigma$ & $130(100)$ & $72(100)$ & & \\
\hline \multirow[t]{12}{*}{ rs2066842 } & All & $\mathrm{TT}$ & $2(1.15)$ & $0(0)$ & $0.852^{\mathrm{b}}$ & 0.644 \\
\hline & & $\mathrm{TC}$ & $65(37.15)$ & $37(40.2)$ & & \\
\hline & & $\mathrm{CC}$ & $108(61.7)$ & $55(59.8)$ & & \\
\hline & & $\Sigma$ & $175(100)$ & $92(100)$ & & \\
\hline & Male & $\mathrm{TT}$ & $1(2.3)$ & $0(0)$ & $0.588^{\mathrm{b}}$ & 0.856 \\
\hline & & $\mathrm{TC}$ & $19(43.2)$ & $8(40)$ & & \\
\hline & & $\mathrm{CC}$ & $24(54.5)$ & $12(60)$ & & \\
\hline & & $\Sigma$ & $44(100)$ & $20(100)$ & & \\
\hline & Female & $\mathrm{TT}$ & $1(0.8)$ & $0(0)$ & $1.011^{\mathrm{b}}$ & 0.706 \\
\hline & & $\mathrm{TC}$ & $46(35.1)$ & $29(40.3)$ & & \\
\hline & & $\mathrm{CC}$ & $84(64.1)$ & $43(59.7)$ & & \\
\hline & & $\Sigma$ & $131(100)$ & $72(100)$ & & \\
\hline
\end{tabular}

${ }^{\mathrm{a} A n a l y s e d ~ u s i n g ~ a ~} \chi^{2}$ test. ${ }^{\mathrm{b}}$ Analysed using a Fischer's exact test.

and IL-17F) in patients with MS, which could additionally be used to differentiate between MS subtypes. Several other studies have also shown evidence of increased pro-inflammatory cytokine production, for example, the macrophage migration inhibitory factor (MIF) family of cytokines, and its homolog MIF 2 in patients with MS $(26,27)$. On the other hand, the production of endogenous anti-inflammatory cytokines and their antagonists (IL-37, IL-1 receptor antagonist, heme oxygenase, amongst others) seems to be reduced in patients with MS (28-30). This indicates that future studies are warranted to reveal the complex immunological pathways involved in MS pathogenesis.

The role of NOD2 polymorphisms in the pathogenesis of MS and response to IFN- $\beta$ therapy is most likely only one part of the complex multidirectional system of genetic, immunological and environmental factors yet to be elucidated. Considering the major role PRRs serve in our immune system, a connection between NOD2 and MS seemed promising and worth exploring, since little research has been published on this topic so far $(9,10)$. Since PRRs are directly involved in our immune response to foreign molecules, it seemed prudent to explore the potential association between the NOD2 and IFN- $\beta$ treatment response in MS.
The limitation of the relatively small sample size in the present study should be mentioned. Yet, this is a representative sample from the local region, and thus represents a true NOD2 rs2066842 polymorphism association with susceptibility to MS. Furthermore, given that no studies have performed a detailed haplotype-based analysis of NOD2 in patients with MS, to the best of our knowledge, the possibility of allelic heterogeneity cannot be ruled out. A possible MS risk gene could exist and be in linkage disequilibrium with the NOD2 rs2066842 allele.

\section{Acknowledgements}

Not applicable.

\section{Funding}

No funding was received.

\section{Availability of data and materials}

The datasets used and/or analysed during the present study are available from the corresponding author on reasonable request. 


\section{Authors' contributions}

AZ, AM, SR, NSC, BP and LL prepared the materials, and collected ana analysed the data. AZ and LL wrote the manuscript, and all authors revised the manuscript. All authors have read and approved the final manuscript. AZ and LL confirm the authenticity of the raw data.

\section{Ethics approval and consent to participate}

The present study was approved by the Ethical Committees of both participating centres and all subjects provided written informed consent to participate.

\section{Patient consent for publication}

Not applicable.

\section{Competing interests}

The authors declare that they have no competing interests.

\section{References}

1. Compston A and Coles A: Multiple sclerosis. Lancet 359: 1221-1231, 2002

2. Bertolotto A: Neutralizing antibodies to interferon beta: Implications for the management of multiple sclerosis. Curr Opin Neurol 17: 241-246, 2004.

3. Wingerchuk DM and Weinshenker BG: Disease modifying therapies for relapsing multiple sclerosis. BMJ 354: i3518, 2016.

4. PRISMS Study Group and the University of British Columbia MS/MRI Analysis Group: PRISMS-4: Long-term efficacy of interferon-beta-1a in relapsing MS. Neurology 56: 1628-1636, 2001.

5. Ebers GC; PRISM Study Group: Randomised double-blind placebo-controlled study of interferon $\beta$-1a in relapsing/remitting multiple sclerosis. PRISMS (Prevention of Relapses and Disability by Interferon $\beta$-1a Subcutaneously in Multiple Sclerosis) Study Group. Lancet 352: 1498-1504, 1998.

6. Enevold C, Oturai AB, Sørensen PS, Ryder LP, Koch-Henriksen N and Bendtzen K: Polymorphisms of innate pattern recognition receptors, response to interferon-beta and development of neutralizing antibodies in multiple sclerosis patients. Mult Scler 16: 942-949, 2010.

7. The IFNB Multiple Sclerosis Study Group and the University of British Columbia MS/MRI Analysis Group: Neutralizing antibodies during treatment of multiple sclerosis with interferon beta-1b: Experience during the first three years. Neurology 47: 889-894, 1996

8. Sorensen PS, Ross C, Clemmesen KM, Bendtzen K, Frederiksen JL, Jensen K, Kristensen O, Petersen T, Rasmussen S, Ravnborg M, et al; Danish Multiple Sclerosis Study Group: Clinical importance of neutralising antibodies against interferon beta in patients with relapsing-remitting multiple sclerosis. Lancet 362: 1184-1191, 2003.

9. Hedegaard CJ, Enevold C, Sellebjerg F, Bendtzen K and Nielsen $\mathrm{CH}$ : Variation in NOD2 augments Th2- and Th17 responses to myelin basic protein in multiple sclerosis. PLoS One 6: e20253, 2011.

10. Enevold C, Oturai AB, Sørensen PS, Ryder LP, Koch-Henriksen N and Bendtzen K: Multiple sclerosis and polymorphisms of innate pattern recognition receptors TLR1-10, NOD1-2, DDX58, and IFIH1. J Neuroimmunol 212: 125-131, 2009.

11. McDonald WI, Compston A, Edan G, Goodkin D, Hartung HP, Lublin FD, McFarland HF, Paty DW, Polman CH, Reingold SC, et al: Recommended diagnostic criteria for multiple sclerosis: Guidelines from the International Panel on the diagnosis of multiple sclerosis. Ann Neurol 50: 121-127, 2001.

12. Río J, Nos C, Tintoré M, Téllez N, Galán I, Pelayo R, Comabella M and Montalban X: Defining the response to interferon-beta in relapsing-remitting multiple sclerosis patients. Ann Neurol 59: 344-352, 2006
13. Kurtzke JF: Rating neurologic impairment in multiple sclerosis: An expanded disability status scale (EDSS). Neurology 33: 1444-1452, 1983.

14. Howe KL, Achuthan P, Allen J, Allen J, Alvarez-Jarreta J, Amode MR, Armean IM, Azov AG, Bennett R, Bhai J, et al: Ensembl 2021. Nucleic Acids Res 49: D884-D891, 2021.

15. Karolchik D, Baertsch R, Diekhans M, Furey TS, Hinrichs A, Lu YT, Roskin KM, Schwartz M, Sugnet CW, Thomas DJ, et al: The UCSC genome browser database. Nucleic Acids Res 31: 51-54, 2003.

16. Safran M, Dalah I, Alexander J, Rosen N, Iny Stein T, Shmoish M, Nativ N, Bahir I, Doniger T, Krug H, et al: GeneCards Version 3: the human gene integrator. Database (Oxford) 2010: baq020, 2010.

17. Farré D, Roset R, Huerta M, Adsuara JE, Roselló L, Albà MM and Messeguer X: Identification of patterns in biological sequences at the ALGGEN server: PROMO and MALGEN. Nucleic Acids Res 31: 3651-3653, 2003.

18. 1000 Genomes Project Consortium, Abecasis GR, Auton A, Brooks LD, DePristo MA, Durbin RM, Handsaker RE, Kang HM, Marth GT and McVean GA: An integrated map of genetic variation from 1,092 human genomes. Nature 491: 56-65, 2012.

19. Xu Z and Taylor JA: SNPinfo: integrating GWAS and candidate gene information into functional SNP selection for genetic association studies. Nucleic Acids Res 37 (Web Server issue): W600-W605, 2009.

20. Court MH: A simple calculator to determine whether observed genotype frequencies are consistent with Hardy-Weinberg equilibrium. 2008. https://accounts.smccd.edu/case/biol215/docs/ HW_calculator.xls

21. Royal W III, Gartner S and Gajewski CD: Retinol measurements and retinoid receptor gene expression in patients with multiple sclerosis. Mult Scler 8: 452-458, 2002.

22. Hoffmann S, Cepok S, Grummel V, Lehmann-Horn K, Hackermüller J, Stadler PF, Hartung HP, Berthele A, Deisenhammer F, Wassmuth R, et al: HLA-DRB1*0401 and HLA-DRB $1 * 0408$ are strongly associated with the development of antibodies against interferon-beta therapy in multiple sclerosis. Am J Hum Genet 83: 219-227, 2008.

23. Buck D, Cepok S, Hoffmann S, Grummel V, Jochim A, Berthele A, Hartung HP, Wassmuth R and Hemmer B: Influence of the HLA-DRB1 genotype on antibody development to interferon beta in multiple sclerosis. Arch Neurol 68: 480-487, 2011.

24. Barbosa MD, Vielmetter J, Chu S, Smith DD and Jacinto J: Clinical link between MHC class II haplotype and interferon-beta (IFN-beta) immunogenicity. Clin Immunol 118: 42-50, 2006.

25. Arellano G, Acuña E, Reyes LI, Ottum PA, De Sarno P, Villarroel L, Ciampi E, Uribe-San Martín R, Cárcamo C and Naves R: Th1 and Th17 cells and associated cytokines discriminate among clinically isolated syndrome and multiple sclerosis phenotypes. Front Immunol 8: 753, 2017.

26. Fagone P, Mazzon E, Cavalli E, Bramanti A, Petralia MC, Mangano K, Al-Abed Y, Bramati P and Nicoletti F: Contribution of the macrophage migration inhibitory factor superfamily of cytokines in the pathogenesis of preclinical and human multiple sclerosis: In silico and in vivo evidences. J Neuroimmunol 322: 46-56, 2018.

27. Benedek G, Meza-Romero R, Jordan K, Zhang Y, Nguyen H, Kent G, Li J, Siu E, Frazer J, Piecychna M, et al: MIF and D-DT are potential disease severity modifiers in male MS subjects. Proc Natl Acad Sci USA 114: E8421-E8429, 2017.

28. Cavalli E, Mazzon E, Basile MS, Mammana S, Pennisi M, Fagone P, Kalfin R, Martinovic V, Ivanovic J, Andabaka M, et al: In silico and in vivo analysis of IL37 in multiple sclerosis reveals its probable homeostatic role on the clinical activity, disability, and treatment with fingolimod. Molecules 25: 20, 2019.

29. Fagone P, Patti F, Mangano K, Mammana S, Coco M, Touil-Boukoffa C, Chikovani T, Di Marco R and Nicoletti F: Heme oxygenase-1 expression in peripheral blood mononuclear cells correlates with disease activity in multiple sclerosis. J Neuroimmunol 261: 82-86, 2013.

30. Dujmovic I, Mangano K, Pekmezovic T, Quattrocchi C, Mesaros S, Stojsavljevic N, Nicoletti F and Drulovic J: The analysis of IL-1 beta and its naturally occurring inhibitors in multiple sclerosis: The elevation of IL-1 receptor antagonist and IL-1 receptor type II after steroid therapy. J Neuroimmunol 207: 101-106, 2009. 